\title{
The Cold Dark Matter Model with Cosmological Constant and the Flatness Constraint
}

\author{
A.C.B. Antunes* \\ Instituto de Física, Universidade Federal do Rio de Janeiro \\ C.P. 68528, Ilha do Fundão, 21945-970 Rio de Janeiro, RJ, Brazil \\ L.J. Antunes ${ }^{\dagger}$ \\ Instituto de Engenharia Nuclear - CNEN C.P. 68550, \\ Ilha do Fundão, 21945-970 Rio de Janeiro, RJ, Brazil \\ (Received on 29 January, 2009)
}

\begin{abstract}
The Hubble parameter, a function of the cosmological redshift, is derived from the Friedmann-RobertsonWalker equation. The three physical parameters $H_{0}, \Omega_{0 m}$ and $\Omega_{\Lambda}$ are determined fitting the Hubble parameter to the data from measurements of redshift and luminosity distances of type-Ia supernovae. The best fit is not consistent with the flatness constraint $(k=0)$. On the other hand, the flatness constraint is imposed on the Hubble parameter and the physical parameters used are the published values of the standard model of cosmology. The result is shown to be inconsistent with the data from type-Ia supernovae.
\end{abstract}

Keywords: Cold dark matter model, Hubble parameter.

\section{THE HUBBLE PARAMETER FROM THE FRIEDMANN EQUATION}

From Einstein's equations for the gravitational field in the Robertson-Walker metric, one can derive the Friedmann differential equation

$$
\frac{\dot{R}^{2}}{c^{2} R^{2}}+\frac{k}{R^{2}}-\frac{\Lambda}{3}=\frac{8 \pi G \rho_{m}}{3 c^{2}}
$$

and the acceleration equation

$$
\frac{\ddot{R}^{2}}{c^{2} R}=-\frac{4 \pi G}{3 c^{2}}\left(\rho_{m}+\frac{3 p}{c^{2}}\right)+\frac{1}{3} \Lambda
$$

where $R$ is the scale factor, $k$ is the curvature index and $\Lambda$ the cosmological constant. The pressure $p$ is related to the matter density $\rho_{m}$ by an equation of state,

$$
p=w c^{2} \rho_{m}
$$

with $w=0$ for non-relativistic matter [1-4].

Using the vacuum energy density

$$
\rho_{\Lambda}=c^{2} \Lambda / 8 \pi G
$$

and introducing the Hubble parameter

$$
H(R)=\frac{\dot{R}}{R}
$$

the Friedmann equation reads :

$$
H^{2}(R)+\frac{c^{2} k}{R^{2}}=\frac{8 \pi G}{3}\left(\rho_{m}+\rho_{\Lambda}\right) .
$$

The scale factor $R$ and the matter density $\rho_{m}$ are related to their present day values $R_{0}$ and $\rho_{0 m}$ by

$$
\rho_{m} R^{3}=\rho_{0 m} R_{0}^{3}
$$

\footnotetext{
*Electronic address: antunes@if.ufrj.br

†Electronic address: leila@ien.gov.br
}

Defining an adimensional variable, the cosmological frequency redshift,

$$
x=\frac{R_{0}}{R}=1+z
$$

where $z$ is the redshift, the equation above becomes

$$
H^{2}(x)+\frac{c^{2} k}{R_{0}^{2}} x^{2}=\frac{8 \pi G}{3}\left(\rho_{0 m} x^{3}+\rho_{\Lambda}\right) .
$$

For current values, corresponding to $x=1$, this equation gives

$$
\frac{c^{2} k}{R_{0}^{2}}=\frac{8 \pi G}{3}\left(\rho_{0 m}+\rho_{\Lambda}-\rho_{c}\right),
$$

where $\rho_{c}=\left(3 H_{0}^{2} / 8 \pi G\right)$ is the critical density and $H_{0}$ is the Hubble constant.

Now the Hubble parameter can be written explicitly as

$$
H^{2}(x)=\frac{8 \pi G}{3}\left[\rho_{\Lambda}-\left(\rho_{0 m}+\rho_{\Lambda}-\rho_{c}\right) x^{2}+\rho_{0 m} x^{3}\right] .
$$

Introducing the relative densities $\Omega_{0 m}=\rho_{0 m} / \rho_{c}$ and $\Omega_{\Lambda}=$ $\rho_{\Lambda} / \rho_{c}$, the Hubble parameter reads

$$
H^{2}(x)=H_{0}^{2}\left[\Omega_{\Lambda}-\left(\Omega_{0 m}+\Omega_{\Lambda}-1\right) x^{2}+\Omega_{0 m} x^{3}\right] .
$$

The function containing the curvature index and the present day scale factor becomes

$$
\frac{c^{2} k}{R_{0}^{2}}=H_{0}^{2}\left(\Omega_{0 m}+\Omega_{\Lambda}-1\right) .
$$

The acceleration equation

$$
\frac{\ddot{R}}{R}=-\frac{4 \pi G}{3}\left(\rho_{m}+\frac{3 p}{c^{2}}\right)+\frac{c^{2} \Lambda}{3}
$$

can be rewritten as

$$
\frac{\ddot{R}}{R}=H_{0}^{2}\left[\Omega_{\Lambda}-\frac{1}{2}\left(\Omega_{0 m} x^{3}+\frac{3 p}{c^{2} \rho_{c}}\right)\right] .
$$


The left-hand side can be written in terms of $x=R_{0} / R$ and $H(R)=\dot{R} / R$. Using

$$
R \frac{d}{d R}\left(H^{2}\right)=-x \frac{d}{d x}\left(H^{2}\right)
$$

in

$$
\frac{\ddot{R}}{R}=H^{2}(R)+\frac{R}{2} \frac{d}{d R}\left(H^{2}(R)\right)
$$

we obtain

$$
\frac{\ddot{R}}{R}=H^{2}(x)-\frac{x}{2} \frac{d}{d x} H^{2}(x) .
$$

Performing the calculation of the right-hand side with

$$
H^{2}(x)=H_{0}^{2}\left[\Omega_{\Lambda}-\left(\Omega_{0 m}+\Omega_{\Lambda}-1\right) x^{2}+\Omega_{0 m} x^{3}\right]
$$

and equating to the above expression for $\ddot{R} / R$ containing $\left(3 p / c^{2} \rho_{c}\right)$ we obtain $p=0$. Thus, the acceleration equation is finally reduced to

$$
\frac{\ddot{R}}{R}=H_{0}^{2}\left[\Omega_{\Lambda}-\frac{1}{2} \Omega_{0 m} x^{3}\right] .
$$

This result permits to obtain the value of $x$ at the equilibrium point corresponding to $\ddot{R}=0$ :

$$
x_{e}=\left(2 \Omega_{\Lambda} / \Omega_{0 m}\right)^{1 / 3} .
$$

The dimensionless deceleration parameter

$$
q=-\frac{R \ddot{R}}{\dot{R}^{2}}=-\frac{\ddot{R}}{R H^{2}}
$$

can be calculated at the present day condition $(x=1)$ :

$$
q_{0}=-\frac{1}{H_{0}^{2}}\left(\frac{\ddot{R}}{R}\right)_{0}=-\left(\Omega_{\Lambda}-\frac{1}{2} \Omega_{0 m}\right)
$$

The age of the universe $\left(t_{0}\right)$ can be obtained from the Hubble parameter

$$
H(R)=\frac{\dot{R}}{R}=-\frac{1}{x} \frac{d x}{d t},
$$

then

$$
t_{0}=\int_{1}^{\infty} \frac{d x}{x H(x)}
$$

With the above expression for $H^{2}(x)$ we have

$$
H_{0} t_{0}=\int_{1}^{\infty} \frac{d x}{x \sqrt{\Omega_{\Lambda}-\left(\Omega_{0 m}+\Omega_{\Lambda}-1\right) x^{2}+\Omega_{0 m} x^{3}}}
$$

\subsection{Determination of the parameters by fitting $H(x)$ to type-Ia supernovae data}

Let $\widetilde{H}_{0}=65 \mathrm{~km} \cdot \mathrm{s}^{-1} \cdot \mathrm{Mpc}^{-1}$ be a nominal value of the Hubble constant; defining the function

$$
y(x)=\frac{H^{2}(x)}{\widetilde{H}_{0}^{2}},
$$

with

$$
y_{0}=\frac{H_{0}^{2}}{\widetilde{H}_{0}^{2}},
$$

and also the parameters

$$
a_{1}=y_{0} \Omega_{\Lambda}, a_{2}=-y_{0}\left(\Omega_{0 m}+\Omega_{\Lambda}-1\right) \text { and } a_{3}=y_{0} \Omega_{0 m} \text {, }
$$

the above equation for the Hubble parameter gives

$$
y(x)=a_{1}+a_{2} x^{2}+a_{3} x^{3} .
$$

The three parameters of this polynomial function can be determined by fitting data from measurements of the luminosity distances and the redshift of the type-Ia supernovae. These parameters and their sum, $y_{0}=a_{1}+a_{2}+a_{3}$, give the physical parameters

$$
\Omega_{0 m}=\frac{a_{3}}{y_{0}}, \quad \Omega_{\Lambda}=\frac{a_{1}}{y_{0}}, \quad \text { and } \quad H_{0}=\widetilde{H}_{0} \sqrt{y_{0}} .
$$

To fit the Hubble parameter to the data from redshift $(z)$ and luminosity distances $(D)$ measurements of type-Ia supernovae, some changes of variables are in order. The published data set is[5]

with $N=230$.

$$
\begin{aligned}
& \left\{z_{j}, \quad v_{j}=\log \left(c z_{j}\right), \quad u_{j}=\log \left(\widetilde{H}_{0} D_{j}\right),\right. \\
& \left.\sigma_{u_{j}} ; j=1, . ., N\right\},
\end{aligned}
$$

The Hubble parameter is

$$
H(x)=(c z / D) .
$$

As

$$
y(x)=\left(H(x) / \widetilde{H}_{0}\right)^{2}=\left(c z / \widetilde{H}_{0} D\right)^{2},
$$

and

$$
\log (y(x))=2\left[\log (c z)-\log \left(\widetilde{H}_{0} D\right)\right]=2(v-u),
$$

then

$$
y(x)=10^{2(v-u)} .
$$

The data set to be used in the fitting is

$$
\left\{x_{j}, y_{j}, \sigma_{j} ; j=1, . ., n\right\}
$$

which is obtained from the first set using

$x_{j}=1+z_{i}, \quad y_{j}=10^{2\left(v_{j}-u_{j}\right)} \quad, \quad$ and $\quad \sigma_{j}=2 \cdot \ln 10 \cdot y_{j} \sigma_{u_{j}}$.

Details of the fitting are presented in the appendix. The results of the fitting give the following values for the physical parameters:

$$
\begin{aligned}
H_{0} & =(60.2 \pm 0.4) \mathrm{km} \cdot \mathrm{s}^{-1} \cdot \mathrm{Mpc}^{-1} \\
\Omega_{0 m} & =0.26 \pm 0.04 \\
\Omega_{\Lambda} & =1.55 \pm 0.11 \\
q_{0} & =-1.42 \pm 0.02
\end{aligned}
$$


and the age of the universe

$$
t_{0}=(30.0 \pm 1.3) \times 10^{9} \mathrm{yr}
$$

The point of null acceleration is

$$
x_{e}=\left(2 \frac{\Omega_{\Lambda}}{\Omega_{o m}}\right)^{1 / 3}=2.28
$$

which corresponds to the redshift

$$
z_{e}=1.28
$$

\section{THE FLATNESS CONSTRAINT}

The cosmic microwave background (CMB) observations suggest that the spacial geometry of the Universe is very close to flat. According to equation (13) the zero curvature corresponds to the condition $\Omega_{o m}+\Omega_{\Lambda}=1$. If this condition is imposed on the Hubble parameter (see equation (12)), we have

$$
H^{2}(x)=H_{0}^{2}\left[\Omega_{\Lambda}+\Omega_{0 m} x^{3}\right]
$$

with

$$
\Omega_{\Lambda}=1-\Omega_{0 m}
$$

The polynomial form, equation (26), becomes

$$
y(x)=a_{1}+a_{3} x^{3}
$$

where $y(x)=\left(H(x) / \widetilde{H}_{0}\right)^{2}, a_{1}=y_{0} \Omega_{\Lambda}, a_{3}=y_{0} \Omega_{0 m}$ and $y_{0}=\left(H_{0} / \widetilde{H}_{0}\right)^{2}$.

The accepted values for the physical parameters of the cold dark matter model with cosmological constant $(\Lambda \mathrm{CDM})$ subjected to the flatness constraint $(k=0)$ are [6]:

$$
H_{0}=71 \pm 4 \mathrm{~km} \cdot \mathrm{s}^{-1} \cdot \mathrm{Mpc}^{-1} \text { and } \Omega_{\Lambda}=0.73 \pm 0.004 \text {. }
$$

So, the coefficients of the polynomial in equation (32) are $a_{1}=0.87$ and $a_{3}=0.32$. The deceleration parameter is

$$
q_{0}=-\frac{1}{2}\left(3 \Omega_{\Lambda}-1\right)=-0.6
$$

and the point of null acceleration is

$$
x_{e}=\left(\frac{2 \Omega_{\Lambda}}{\Omega_{0} m}\right)^{1 / 3}=1.8,
$$

corresponding to the redshift $z_{e}=0.8$. The age of the Universe is

$$
t_{0}=13.7 \mathrm{Gyr} .
$$

The sum of the weighted square deviations for this model, as defined in the appendix, is

$$
\chi^{2}=16780.10
$$

\section{CONCLUSION}

The cold dark matter model with cosmological constant $(\Lambda C D M)$ is expressed by the Hubble parameter as a function of the cosmological redshift $x=1+z$. This function is derived from the Friedmann equation in the Robertson-Walker metric. The square of the Hubble parameter is an incomplete third-degree polynomial function in the variable $x=1+z$. This polynomial is least-squares fitted to data from the measurements of the redshifts and luminosity distances of the type-Ia supernovae, and the three non-null coefficients of the polynomial and the uncertainties and covariances are then computed. The physical parameters are obtained from the three non-null coefficients, showing that these supernovae data are sufficient to determine $H_{0}, \Omega_{0 m}$ and $\Omega_{\Lambda}$, the three fundamental parameters of the $\Lambda C D M$ model. The results of this model are compared with the published results of the $\Lambda C D M$ model with the flatness constraint $(k=0)[6]$ (see Fig. $1)$.

In this second model, the $\Lambda C D M(k=0)$, the measurements from the cosmic microwave background (CMB) are also taken into account. The results of these models disagree. The $\Lambda C D M$ model fitted to the Ia supernovae data implies a positive curvature index $(k=+1)$ and a large age for the Universe. This conflicts with the results of the CMB measurements. On the other hand, the $\Lambda C D M(k=0)$ model presents large deviations from the type-Ia supernovae data. Both models are consistent with the evidences of an accelerating expansion of the Universe [7-11]. However, in any way there are clear disagreements between models and data. Some observable measurements are model-dependent. These observables are related to the parameters of the model. The values of the parameters must be fixed so that these observables can be computed from other observable measurements.

Thus, it is a contradictory result that the $\Lambda C D M(k=0)$ model, which is used in the computation of the luminosity distances of these Ia supernovae, be in disaccord with these same data.

\section{Appendix A}

In this appendix we collect some formulas used in the fitting of the polynomial function

$$
\begin{aligned}
f\left(x ; a_{1}, a_{2}, a_{3}\right) & =a_{1} f_{1}(x)+a_{2} f_{2}(x)+a_{3} f_{3}(x), \\
\text { with } f_{1}(x) & =1, \\
f_{2}(x) & =x^{2}, \text { and } f_{3}(x)=x^{3}
\end{aligned}
$$

to the data set

$$
\left\{x_{j}, y_{j}, \sigma_{j} ; j=1, . ., n\right\}
$$

obtained from the published data[5] by the transformations

$$
x_{j}=1+z_{i} \quad y_{j}=10^{2\left(v_{j}-u_{j}\right)} \quad \sigma_{j}=2 \cdot \ln 10 \cdot y_{j} \cdot \sigma_{u_{j}} .
$$




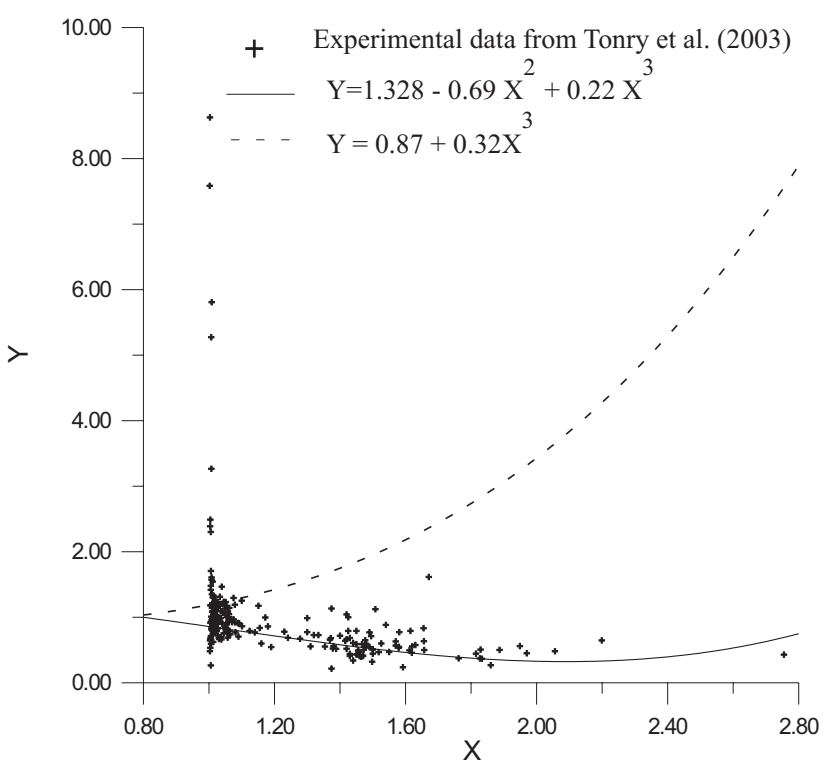

FIG. 1: A plot of the function $y(x)=\left(H(x) / \widetilde{H}_{0}\right)^{2}$ for two models: the best fit for the $\Lambda C D M$ model with $k=+1$ (continuous line) and the $\Lambda C D M$ with $k=0$ with the published values[6] for the physical parameters (dashed line). For comparison, the experimental points corresponding to $\left\{x_{j}, y_{j}\right\}[5]$ are also depicted.

The least-squares method is used to determine the parameters $a_{1}, a_{2}$ and $a_{3}$ which minimize the function

$\chi^{2}\left(a_{1}, a_{2}, a_{3}\right)=\sum_{j=1}^{N} p_{j}\left[y_{j}-f\left(x_{j} ; a_{1}, a_{2}, a_{3}\right)\right]^{2}$, where $\quad p_{j}=\frac{1}{\sigma_{j}}$.

The minimum condition

$$
\frac{\partial \chi^{2}}{\partial a_{j}}=0 \quad(j=1,2,3)
$$

gives a matricial equation

$$
\mathbf{M A}=\mathbf{B},
$$

where

$$
\begin{aligned}
\mathbf{A} & =\left(a_{1}, a_{2}, a_{3}\right)^{\top}, \\
M_{j, k} & =\sum_{i=1}^{N} p_{i} f_{j}\left(x_{i}\right) f_{k}\left(x_{i}\right), \\
B_{j} & =\sum_{i=1}^{N} p_{i} y_{i} f_{j}\left(x_{i}\right), \quad(j, k=1,2,3) .
\end{aligned}
$$

Inverting the matricial equation above, the parameters are given by

$$
a_{j}=\sum_{k=1}^{3}\left(M^{-1}\right)_{j k} B_{k}
$$

The uncertainties and covariances are respectively

and

$$
\sigma_{a j}=\sqrt{\left(M^{-1}\right)_{j j}}
$$

$$
\sigma_{a j k}=\operatorname{cov}\left(a_{j}, a_{k}\right)=\left(M^{-1}\right)_{j k}
$$

The results of the fitting are

$$
\begin{aligned}
a_{1} & =1.328 \pm 0.042 \\
a_{2} & =-0.690 \pm 0.061 \\
a_{3} & =0.220 \pm 0.026 \\
\sigma_{a 12} & =-0.00242 \\
\sigma_{a 13} & =0.00098 \\
\sigma_{a 23} & =-0.00155 .
\end{aligned}
$$

The sum of the weighted square deviations $\left(\chi^{2}\right)$ defined above is

$$
\chi^{2}=989.4
$$

The physical parameters are given by

$$
\begin{aligned}
y_{0} & =a_{1}+a_{2}+a_{3}, \\
H_{0} & =\widetilde{H}_{0} \sqrt{y_{0}}, \\
\Omega_{0 m} & =\frac{a_{3}}{y_{0}}, \\
\Omega_{\Lambda} & =\frac{a_{1}}{y_{0}}
\end{aligned}
$$

and

$$
q_{0}=\frac{\left(\frac{1}{2} a_{3}-a_{1}\right)}{y_{0}}
$$

The uncertainties in these parameters are respectively 


$$
\begin{aligned}
\sigma_{y_{0}}= & \left\{\sigma_{a_{1}}^{2}+\sigma_{a_{2}}^{2}+\sigma_{a_{3}}^{2}+2\left(\sigma_{a_{12}}+\sigma_{a_{13}}+\sigma_{a_{23}}\right)\right\}^{1 / 2}, \\
\sigma_{H_{0}}= & \frac{1}{2} \widetilde{H}_{0} \frac{\sigma_{y_{0}}}{\sqrt{y_{0}}} \\
\sigma_{\Omega_{\Lambda}}= & \frac{1}{y_{0}^{2}}\left\{\left(a_{2}+a_{3}\right)^{2} \sigma_{a_{1}}^{2}+a_{1}^{2} \sigma_{a_{2}}^{2}+a_{1}^{2} \sigma_{a_{3}}^{2}+2 a_{1}^{2} \sigma_{a_{23}}-2 a_{1}\left(a_{2}+a_{3}\right)\left(\sigma_{a_{12}}+\sigma_{a_{13}}\right)\right\}^{1 / 2} \\
\sigma_{\Omega_{0 m}}= & \frac{1}{y_{0}^{2}}\left\{\left(a_{1}+a_{2}\right)^{2} \sigma_{a_{3}}^{2}+a_{3}^{2} \sigma_{a_{1}}^{2}+a_{3}^{2} \sigma_{a_{2}}^{2}+2 a_{3}^{2} \sigma_{a_{12}}-2 a_{3}\left(a_{1}+a_{2}\right)\left(\sigma_{a_{13}}+\sigma_{a_{23}}\right)\right\}^{1 / 2} \\
\sigma_{q_{0}}= & \frac{1}{2 y_{0}^{2}}\left\{\left(2 a_{2}+3 a_{3}\right)^{2} \sigma_{a_{1}}^{2}+\left(a_{3}-2 a_{1}\right)^{2} \sigma_{a_{2}}^{2}+\left(3 a_{1}+a_{2}\right)^{2} \sigma_{a_{3}}^{2}+2\left(2 a_{2}+3 a_{3}\right)\left(a_{3}-2 a_{1}\right) \sigma_{a_{12}}\right. \\
& \left.-2\left(2 a_{2}+3 a_{3}\right)\left(3 a_{1}+a_{2}\right) \sigma_{a_{13}}-2\left(a_{3}-2 a_{1}\right)\left(3 a_{1}+a_{2}\right) \sigma_{a_{23}}\right\}^{1 / 2}
\end{aligned}
$$

The integral that gives the age of the universe is

The uncertainty in $t_{0}$ is

$$
K\left(a_{1}, a_{2}, a_{3}\right)=\left(t_{0} \widetilde{H}_{0}\right)=\int_{1}^{\infty}\left[a_{1} x^{2}+a_{2} x^{4}+a_{3} x^{5}\right]^{-1 / 2} d x .
$$

In order to obtain the uncertainty in $K$ we must compute the derivatives $K_{j}^{\prime}=\left(\partial K / \partial a_{j}\right)$ so that $\sigma_{K}$ is given by

$$
\begin{aligned}
\sigma_{K} & =\left(K_{1}^{\prime 2} \sigma_{a_{1}}^{2}+K_{2}^{\prime 2} \sigma_{a_{2}}^{2}+K_{3}^{\prime 2} \sigma_{a_{3}}^{2}+2 K_{1}^{\prime} K_{2}^{\prime} \sigma_{a_{12}}+\right. \\
& \left.+2 K_{1}^{\prime} K_{3}^{\prime} \sigma_{a_{13}}+2 K_{2}^{\prime} K_{3}^{\prime} \sigma_{a_{23}}\right)^{1 / 2}
\end{aligned}
$$

$$
\sigma_{t_{0}}=\frac{\sigma_{K}}{\widetilde{H}_{0}}
$$

The numerical results are

$$
\begin{aligned}
& K=1.992, K_{1}^{\prime}=-1.7204, \\
& K_{2}^{\prime}=-7.1717, K_{3}^{\prime}=-16.652 \\
& \text { and } \sigma_{K}=0.088 .
\end{aligned}
$$

[1] Weinberg, S., Gravitation and Cosmology Principles and Applications of the General Theory of Relativity, (John Wiley and Sons, 1972).

[2] Rindler,W. , Relativity, Special, General and Cosmological, (Oxford University Press, 2001).

[3] D'Inverno, R., Introducing Einstein's Relativity, (Clarendon Press, Oxford, 1998).

[4] Grupen, C. Astroparticle Physics, (Springer, 2005).

[5] Tonry, J.L., et al., Astro-ph/0305008, Vol. 1 (2003).
[6] Particle Data Group, Review of Particle Physics, Journal of Physics G, Vol. 33 (July 2006).

[7] Riess, A.G. et al., Astron. J. 116, 1009 (1988).

[8] Perlmutter, S. et al., Astrophy. J. 517(1999), 565 .

[9] Tonry, J.L., et al., Astrophys. J. 594 (2003), 1.

[10] Riess, A.G. et al., Astrophys. J. 607 (2004), 665.

[11] Spergell, D.N. Astrophys. J. Suppl. ser. 170(2007), 377 . 